\title{
Role of Kinase Suppressor of Ras-1 in Lipopolysaccharide-Induced Acute Lung Injury
}

\author{
Xiang Lia Erich Gulbins ${ }^{b}$ Yang Zhang ${ }^{a}$ \\ aDepartment of Pharmacology \& Toxicology, Medical College of Virginia Campus, Virginia \\ Commonwealth University, VA; 'Institute of Molecular Biology, University of Duisburg-Essen, Essen
}

\section{Key Words}

Kinase suppressor of ras-1 1 ERK • Lipopolysaccharide - Acute lung injury

\begin{abstract}
Kinase suppressor of ras-1 (Ksr1) has been recently shown to be a central signaling molecule in the host response to Pseudomonas aeruginosa infections in the lung. Ksr1 functions to regulate the release of nitric oxide (NO)-radicals upon P. aeruginosa infections. Ksr1 also enhances Raf-1/MEK/ERK signaling and is involved in a variety of cellular responses, including cell differentiation, proliferation, and apoptosis. Here, we investigated whether Ksr1 is involved in the host immune response to lipopolysaccharide (LPS), one of the major components of gramnegative bacteria, in the lung. To this end, we induced an acute lung injury in wild type and Ksr1-deficient mice by intratracheal instillation of LPS. We found that LPS-induces acute lung injury, as characterized by cytokine expression, neutrophil infiltration and protein extrusion in wildtype mice. Ksr1-deficient mice showed a very similar reaction to LPS as the wildtype mice. In freshly isolated alveolar macrophages from wild type and Ksr1-deficient mice, LPS increased ERK activation, nuclear translocation of NFKB and expression of inflammatory cytokines and chemokines in a similar pattern. Inhibition of Src or Raf- 1 blocked LPS-induced ERK activation. Taken together, these findings indicate that Ksr1 plays a dispensable role in LPS-induced ERK activation in alveolar macrophages and does not contribute to the development of acute lung injury in the LPS model.
\end{abstract}




\section{Introduction}

Acute lung injury (ALI) and its most severe form, acute respiratory distress syndrome (ARDS), are usually caused by bacterial sepsis of pulmonary and extrapulmonary sources, which ultimately leads to a disruption of alveolar-capillary integrity with severe consequences for pulmonary gas exchange [1]. ALI is an important cause of acute respiratory failure, affecting more than 200,000 patients per year in the United States alone [2]. However, no specific therapy is available. Lipopolysaccharide (LPS) is an essential component of the cell wall of gram-negative bacteria. Exposure of the lower respiratory tract to bacterial LPS by intratracheal instillation is a well-recognized mouse model of ALI and mimics, in many important pathological aspects, the clinical development of ALI induced by gram-negative pulmonary infection [1].

Kinase suppressor of Ras-1 (Ksr1) was first identified in Drosophila melanogaster and Caenorhabditis elegans in 1995 [3]. Ksr1 functions as a ceramide-activated kinase, which phosphorylates and activates Raf-1 to induce Raf-1/MEK/ERK mitogen-activated protein kinase (MAPK) signaling [4]. In addition, Ksr1 can be a scaffold protein that binds Raf-1, MEK, and ERK to facilitate activation of Raf-1/MEK/ERK MAPK signaling [5]. Knockout of the Ksr1 gene results in defects in ERK/MAPK signaling, antigen-triggered T-cell proliferation, formation of hair follicles, Ras-dependent tumor formation, and a reduction of joint damage in rheumatoid arthritis [5-9]. We have previously shown that Ksr1 is critically involved in the host response to Pseudomonas aeruginosa (P. aeruginosa) [10]. Mice deficient for Ksr1 failed to release nitric oxide (NO)-radicals upon pulmonary infection with $P$. aeruginosa leading to a very high sensitivity of Ksr1-deficient mice to pulmonary P. aeruginosa infections. Thus, the present study investigated the role of Ksr1 in a model of LPS-induced ALI in Ksr1-deficient mice, since LPS is one of the major pathogenicity factors of gram-negative bacteria.

Alveolar macrophages, the primary immune cells found in the air spaces, play a central role in promoting alveolar inflammation and the pathogenesis of ALI by secreting proinflammatory cytokines and recruiting polymorphonuclear neutrophils [11-13]. Depletion or dysfunction of alveolar macrophages has been shown to decrease the expression of proinflammatory cytokines and to attenuate ALI [14]. In macrophages, LPS signaling is initiated by an interaction between LPS and LPS-binding protein (LBP); this interaction allows LBP to bind to CD14 and to associate with toll-like receptor 4 (TLR4) leading to activation of TLR4 signaling. Stimulation of TLR4 results in recruitment of myeloid differentiation primary response gene (MyD88), interleukin-1 receptor-associated kinase (IRAK), and tumor necrosis factor (TNF) receptor associated factor 6 (TRAF6) and in activation of the MAPK superfamily cascades and the transcription factors nuclear factor $\kappa B(\mathrm{NF \kappa B})$ and activator protein (AP)-1. These events finally lead to the expression of genes that participate in the innate immune response, including inflammatory cytokines and chemokines. The mechanism of TLR4-mediated activation of ERK is not fully understood. Therefore, we sought to determine whether Ksr1 is involved in ERK activation and the expression of proinflammatory cytokines in the response of freshly isolated alveolar macrophages to LPS.

\section{Materials and Methods}

Murine model of acute lung injury

In isoflurane-anesthetized mice, ALI was induced by intratracheal administration of $30 \mu \mathrm{g}$ LPS (Escherichia coli, 0111:B4, Sigma) in a 20- $\mathrm{LL}$ phosphate buffered saline (PBS) solution. No deaths were associated with intratracheal LPS exposure in this model. ALI, as characterized by infiltration of neutrophils into the lung interstitium, development of interstitial edema, and increased production of proinflammatory cytokines, occurs after the injection of LPS. Previous studies have demonstrated that the greatest accumulation of neutrophils into airways and histologically detectable injury are present $24 \mathrm{~h}$ after LPS exposure [15]. Ksr1-deficient mice (kindly provided by R. Kolesnick) and syngenic wild-type mice (all 8 
to 12 weeks old) were used for all experiments. The local Animal Care and Use Committees approved all procedures.

\section{Immunohistochemistry}

Mice were euthanized, and blood was cleared from the pulmonary circulation by perfusion through

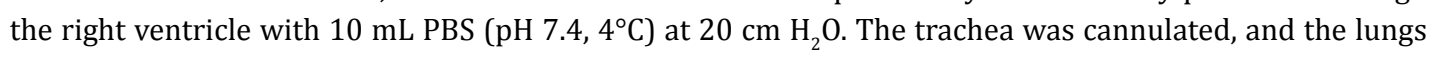
were inflated with $4 \%$ paraformaldehyde (PFA) for 10 minutes at $25 \mathrm{~cm} \mathrm{H}_{2} \mathrm{O}$. The lungs were subsequently removed, fixed in PFA for 24 hours, and embedded in paraffin for sectioning at a thickness of $6 \mu \mathrm{m}$. The sections were stained with hematoxylin and eosin (H\&E).

\section{Wet/dry weight ratios}

All mice used for determination of lung wet/dry weight ratios were of identical ages. Lungs were excised, rinsed briefly in PBS, blotted, and then weighed for determination of wet weight. Lungs were then dried in an oven at $80^{\circ} \mathrm{C}$ for 7 days before determination of dry weight.

Cytokine levels, protein leakage, and neutrophil counts in bronchoalveolar lavage fluid

Bronchoalveolar (BAL) fluid samples were obtained by the following procedure. After the mice were euthanized, a small catheter was inserted into the trachea, and $1 \mathrm{~mL}$ of PBS was injected into the lung and recovered by very gentle suction. This procedure was repeated 5 times, and a total of $5 \mathrm{~mL}$ BAL fluid was collected. For counting of neutrophils in BAL fluid, samples were centrifuged for 5 minutes at $300 \times g$. The resulting pellet was resuspended in $1 \mathrm{~mL}$ PBS with $1 \%$ bovine serum albumin (BSA) and $0.1 \%$ sodium azide, and the total cell number was counted by a hemocytometer. The percentage fraction of polymorphonuclear cells (PMNs) in the BAL fluid was determined by flow cytometry (FACSCalibur, BD Biosciences, Heidelberg, Germany). PMNs were identified by their typical appearance in the forward/side scatter and their expression of Gr1 (PE-anti-Gr1, BD Biosciences).

Appropriate isotype controls (rat IgG2b) were used to compensate for nonspecific antibody binding. Interleukin (IL)-1 $\beta$, TNF- $\alpha$, and the chemokine KCs were quantified in the post-centrifugation supernatants with commercial enzyme-linked immunosorbent assays (ELISAs; R\&D Systems, Abingdon, UK), according to the manufacturer's instructions. The protein concentration in the BAL sample was used as an indicator of the integrity of the blood-pulmonary epithelial cell barrier. Protein concentration in the post-centrifugation supernatants was determined by the Bradford assay (Bio-Rad, Hercules, CA).

\section{Isolation of alveolar macrophages from mice}

Lung macrophages were isolated from BAL fluid obtained as described above. The lung was lavaged with a total of $15 \mathrm{~mL}$ ice-cold PBS in 20 aliquots ( $0.75 \mathrm{~mL}$ per aliquot). Approximately 0.5 to $1 \times 10^{6}$ cells per mouse were consistently obtained. Cells were pelleted by centrifugation at $300 \times g$ for $15 \mathrm{~min}$, resuspended, and cultured for 1 hour in RPMI-1640 (Gibco-Life Technologies, Carlsbad, CA) with 1 mM HEPES (pH, 7.4) in 24-well plates at a density of $10^{5}$ cells per well. Alveolar macrophages are extremely adhesive. Therefore, after other blood cells were washed off, a pure cell culture was obtained in which more than $99 \%$ of cells were macrophages, as confirmed by flow cytometry after staining with fluorescein isothiocyanate (FITC)coupled anti-CD11b antibodies (BD Biosciences).

\section{Immunofluorescence study}

Cells were stimulated with LPS or left unstimulated and then fixed in 2\% PFA/PBS for 10 min. Cells were permeabilized with $0.1 \%$ Triton X-100/PBS for $15 \mathrm{~min}$ at room temperature and washed again with PBS. Nonspecific binding sites were blocked by incubation for 30 min in PBS supplemented with $5 \%$ fetal calf serum. Cells were washed and incubated for $45 \mathrm{~min}$ with $1 \mu \mathrm{g} / \mathrm{mL}$ rabbit anti-p65 antibody (Santa Cruz Biotechnology, Santa Cruz, CA). Cells were then washed in PBS with 0.05\% Tween-20 and incubated for an additional 45 min with Cy3-anti-rabbit immunoglobulin G (IgG) F(ab) ${ }_{2}$ fragment antibodies (Jackson ImmunoResearch, Newmarket, Suffolk, UK) diluted in PBS and 5\% fetal calf serum. After a final wash with PBS, cells were mounted on glass coverslips with Moviol. Control experiments were performed with normal rabbit IgG and secondary antibodies. Control antibodies did not substantially bind to the cells. Cells were examined on a Leica TCS SP5 confocal microscope equipped with a 100×oil objective, and images were analyzed with Leica Confocal software (Leica Microsystems, Wetzlar, Germany). 


\section{Subcellular fractionation}

Microsomal membranes containing plasma membrane and intracellular endomembranes were isolated from the cytosol fraction as previously described [16]. Briefly, $5 \times 10^{6} \mathrm{~J} 774$ macrophages were lysed in $500 \mu \mathrm{L}$ buffer containing $1 \% \mathrm{NP} 40,50 \mathrm{mM}$ Tris-HCl, $1 \mathrm{mM}$ EDTA, and a cocktail of protease inhibitors $(\mathrm{pH}, 7.4)$. Nuclei and cell debris were removed by centrifuging the lysate at $1000 \times g$, and the supernatant was designated as cell homogenate (Homo). Of this cell homogenate, $100 \mu \mathrm{L}$ was reserved for immunoblot analysis. The heavy microsomal membrane (Heavy) fraction was spun down by subjecting the other $400 \mu \mathrm{L}$ of cell homogenate to ultracentrifugation ( $30 \mathrm{~min} ; 40,000 \times \mathrm{g}$ ). The light microsomal membrane (Light) fractions were spun down by subjecting the supernatant fraction containing the cytosolic compartment to further ultracentrifugation $(2 \mathrm{~h}, 100,000 \times \mathrm{g})$, and the supernatant was designated as cytosol. Heavy and light microsomal membrane fractions were resuspended in $400 \mu \mathrm{L}$ lysis buffer, and a $20 \mu \mathrm{L}$ aliquot of each fraction was subjected for immunoblot analysis.

\section{Immunoblot analyses}

ERK phosphorylation in alveolar macrophages was detected by treating cells as indicated and lysing them in sodium dodecyl sulfate (SDS) sample buffer (62.5 mM Tris [pH, 6.8], $10 \%$ glycerol, $2 \%$ SDS, $0.04 \%$ bromophenol blue, and 5\% $\beta$-mercaptoethanol). Chromosomal DNA was broken and sample viscosity was decreased by boiling the cells and sonicating them (3 time for $10 \mathrm{~s}$ each) on ice. Proteins were separated by $10 \%$ SDS polyacrylamide gel electrophoresis (SDS-PAGE) and transferred to nitrocellulose membranes. The blots were blocked in 4\% BSA in Tris-buffered saline supplemented with $0.1 \%$ Tween 20; they were then incubated overnight at $4^{\circ} \mathrm{C}$ with anti-Ksr1 (BD Biosciences), anti-actin (Santa Cruz Biotechnology), and anti-phospho-ERK1/2 (Cell Signaling) or anti-ERK (Cell Signaling) antibodies (all antibodies were diluted 1:1000 in 4\% BSA). Blots were labeled with alkaline phosphatase-coupled secondary antibodies (Santa Cruz Biotechnology) and developed with the Tropix system (Bedford, MA).

\section{Inhibitor studies}

Alveolar macrophages were pretreated with the Raf-1 kinase inhibitor GW5074 (Sigma, Deisenhofen) and with the Src-kinase inhibitor PP2 (Merck-Millipore, Darmstadt) at a concentration of $10 \mu \mathrm{M}$ each for 15 $\min$.

Statistics

Data were subjected to statistical analyses with analysis of variance (ANOVA). Statistical significance was assigned at the level of $\mathrm{P}<0.05$.

\section{Results}

\section{Intratracheal instillation of LPS induces ALI in Ksr 1 \% mice}

To define the role of Ksr1 in the development of ALI, we exposed Ksr1-deficient (Ksr1 ${ }^{\%}$ ) mice and wild-type $\left(\mathrm{Ksr}^{+/+}\right)$controls to intratracheal LPS. As shown in Fig. 1A, histological examinations demonstrate that LPS exposure for $24 \mathrm{~h}$ elicited similar accumulations of inflammatory cells, primarily neutrophils, and increased interstitial edema and leakage of red blood cells into the alveolar spaces in the lungs of wild-type and Ksr1-deficient mice. Lung edema, as determined by the increase in the wet/dry weight ratio of lungs after LPS stimulation, was not significantly different between $\mathrm{Ksr}^{+/+}$and Ksr1\% mice (Fig.1 B).

The number of neutrophils in the BAL fluid was determined by flow cytometric analysis after $24 \mathrm{~h}$ LPS stimulation. LPS dose-dependently increased the number of neutrophils per lung in the BAL samples from Ksr1 ${ }^{+/+}$mice, which were not significantly different from the number in the BAL samples from Ksr ${ }^{\%}$ mice (Fig. 2A). Moreover, LPS dose-dependently increased the severity of lung edema, as determined by evaluating albumin leakage in BAL fluids, in $\mathrm{Ksr}^{+/+}$mice, which also did not differ from Ksr1\% mice; therefore, Ksr1 deficiency had no effect on the LPS-induced increase in BAL protein levels (Fig. 2B).

We also quantified the levels of proinflammatory mediators in BAL fluids after LPS stimulation. The lung cytokines TNF $\alpha$ and IL1 $\beta$ and the chemokine KC, which are derived 
Fig. 1. LPS induces acute lung injury in $\mathrm{Ksr} 1^{+/+}$and $\mathrm{Ksr} 1^{\%}$ mice. Mice were intratracheally treated with $30 \mu \mathrm{g}$ LPS in PBS or PBS only. The lungs were removed after $24 \mathrm{~h}$ and stainedwithhematoxylin/ eosin for visualization of neutrophils or examined for water content. (A) LPS triggers massive influx and accumulation of neutrophils in the lung interstitium in both $\mathrm{Ksr}^{+/+}$and $\mathrm{Ksr}^{\%}$ mice after $24 \mathrm{~h}$ induction. (B) Lung water content after LPS stimulation. Date are presented as mean \pm SD of 6 animals in each group. *** indicates significant differences between mice treated with LPS $v s$ PBS-control treated mice $(\mathrm{P}<0.001)$.

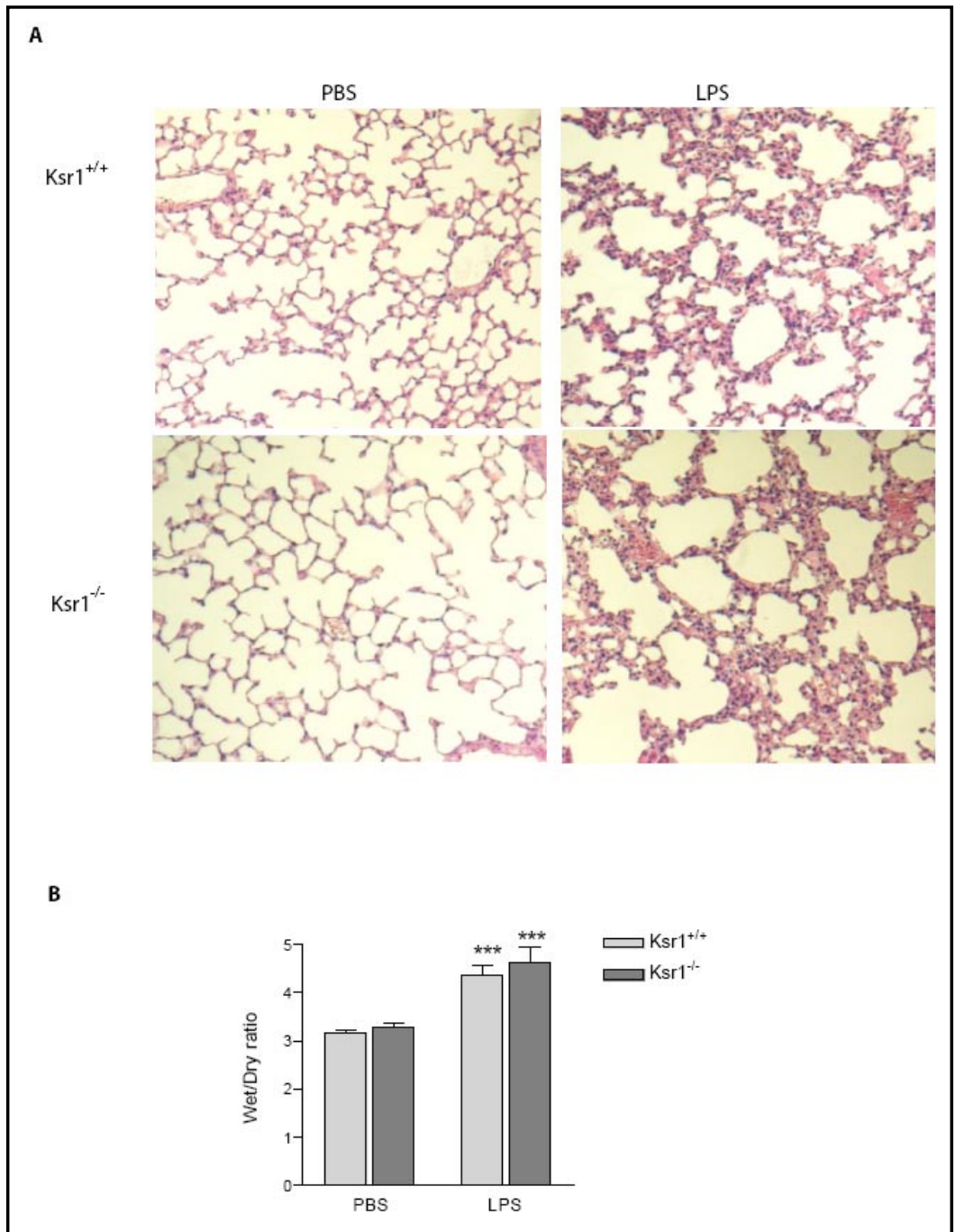

from alveolar macrophages in a NFkB-dependent fashion, are known to play a crucial role in ALI [17]. LPS-induced neutrophil infiltration is dependent on the expression of chemokines (e.g., KC and macrophage-inflammatory protein-2 [MIP-2]). As shown in Fig. 2C, both $\mathrm{Ksr}^{-/}$mice and $\mathrm{Ksr}^{+/++}$mice responded to LPS exposure with a massive increase of cytokines, as shown by analysis of BAL fluids. Collectively, these findings suggest that ALI after airway deposition of LPS is Ksr1-independent.

\section{Expression and localization of Ksr1 in macrophages}

We investigated whether LPS can modulate the subcellular distribution of Ksr1 in macrophages. As shown in Fig. 3, Ksr1 was predominantly detected in the cytosol, whereas much less expression of Ksr1 was detected in Heavy and Light membrane fractions. This pattern was not affected by LPS treatment.

LPS-induced $N F \kappa B$ activation and cytokine production by alveolar macrophages are independent of Ksr1

TLR4 engagement results in the activation of $\mathrm{NF \kappa B}$, a key transcription factor that controls the expression of multiple proinflammatory cytokines and chemokines. Recent studies have demonstrated that Ksr1-mediated MAPK signaling can crosstalk with NFKB activation [18]. In epithelial cells, Ksr1 deficiency results in attenuated NFKB activation and cytokine production [19]. Therefore, we examined whether Ksr1 deficiency affects 
A

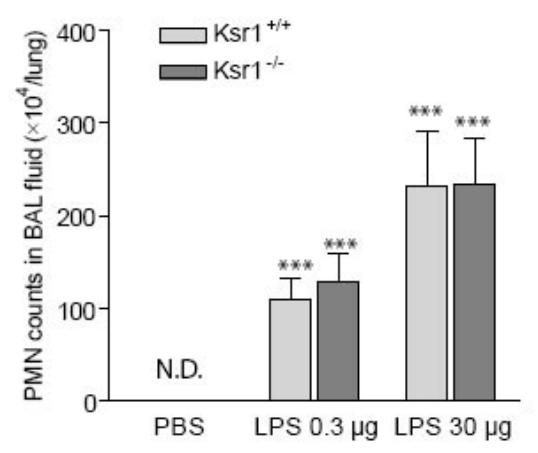

B

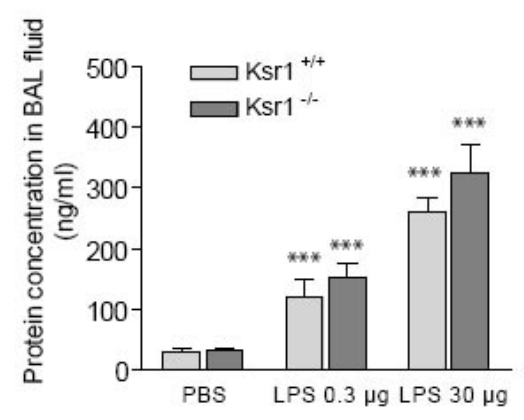

C
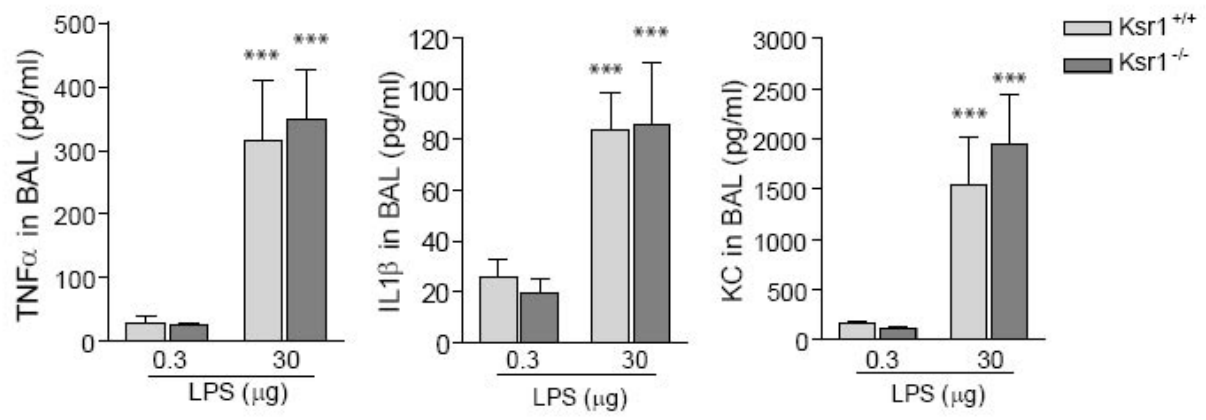

Fig. 2. Ksr1 deficiency has no effects on neutrophil infiltration, protein extrusion, and cytokine levels in lung BAL fluid. Mice were intratracheally treated with LPS, and BAL fluid was collected after 24 hrs. (A) The number of neutrophils in the BAL fluid was determined by flow cytometry with anti-Gr1 antibodies. (B) The protein concentration in BAL fluid was measured by Bradford assay. (C) Levels of TNF $\alpha$, IL1 $\beta$, and the chemokine KC in BAL fluid were determined by enzyme-linked immunosorbent assay (ELISA). Data are presented as mean \pm SD of 6 separate experiments. ${ }^{* * *}$, LPS vs vehicle control $(\mathrm{P}<0.001)$. Abbreviations: BAL, bronchoalveolar lavage; IL, interleukin; Ksr1, kinase suppressor of Ras-1; LPS, lipopolysaccharide; PE, R-phycoerythrin; TNF, tumor necrosis factor.

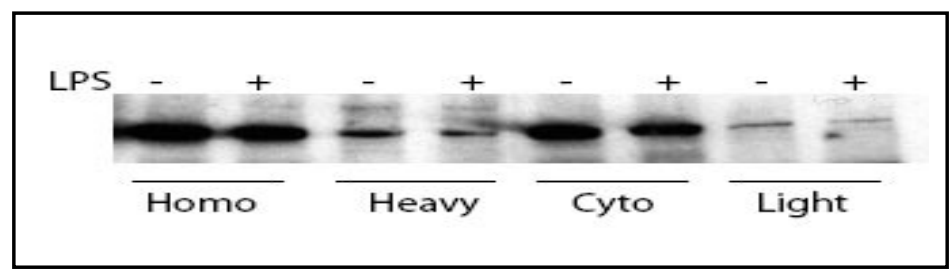

Fig. 3. LPS does not change intracellular expression of Ksr1 in macrophages. Western blots show the localization of Ksr1 in homogenate (Homo), heavy microsomal membrane (Heavy) fraction, cytosol (Cyto), and light microsomal membrane (Light) fraction with or without LPS stimulation (100 ng/mL, $30 \mathrm{~min}$ ). Shown are representative blots from three independent experiments.

LPS-induced NFKB activation in freshly isolated alveolar macrophages. The analysis was performed by determining nuclear translocation of p65, a marker event for NFKB activation. As shown in Fig. 4A, LPS induced a similar nuclear translocation of p65 in both $\mathrm{Ksr}^{+/+}$and $\mathrm{Ksr}^{\%}$ macrophages. Further, $\mathrm{Ksr}^{+/+}$and $\mathrm{Ksr} 1^{\%}$ alveolar macrophages released similar levels of TNF $\alpha$ in response to LPS stimulation (Fig. 4B). 
Fig. 4. LPS induces $\mathrm{NFKB}$ activation and cytokine production in $\mathrm{Ksr} 1^{+/+}$ and $\mathrm{Ksr}^{\%}$ alveolar macrophages. (A) $\mathrm{NF \kappa B}$ activation is independent of Ksr1, Ras, or Raf-1. Freshly isolated alveolar macrophages from wildtype and $\mathrm{Ksr1}^{-/}$mice were treated with LPS (100 ng/mL) or PBS for 30 minutes, permeabilized, and stained with Cy3-labeled anti-p65 antibodies. $\mathrm{NF \kappa B}$ activation was evaluated by nuclear detection of p65 subunits of $\mathrm{NF \kappa B}$. Shown are representative fluorescence microscopy studies from four independent experiments. (B) Time-dependent release of cytokines by alveolar macrophages in response to LPS stimulation (100 ng/mL). Cells were incubated in $1 \mathrm{~mL}$ PBS, and an aliquot $(50 \mu \mathrm{L})$ of supernatant was used for enzyme-linked immunosorbent assay (ELISA) to detect TNF $\alpha$ release. Data are presented as mean \pm SD of four independent experiments. ***, LPS vs vehicle control $(\mathrm{P}<0.001)$.
A

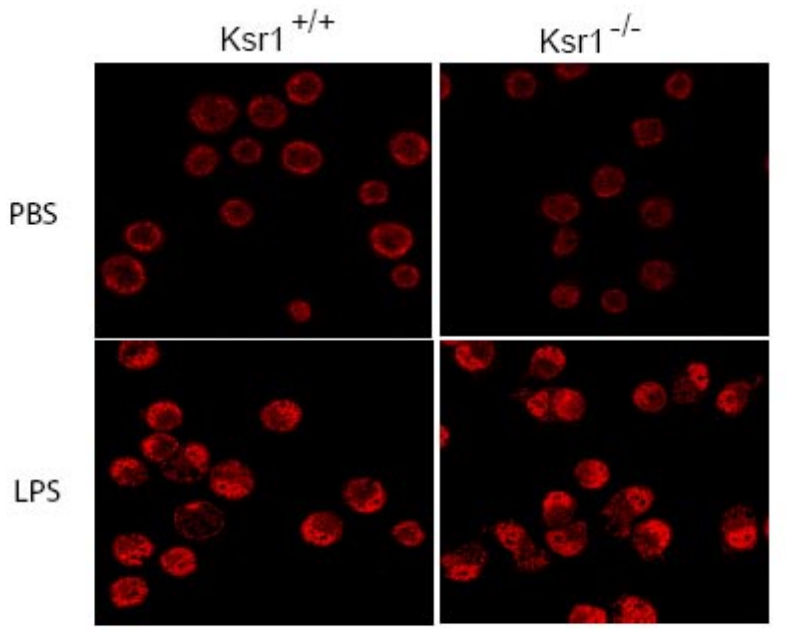

B

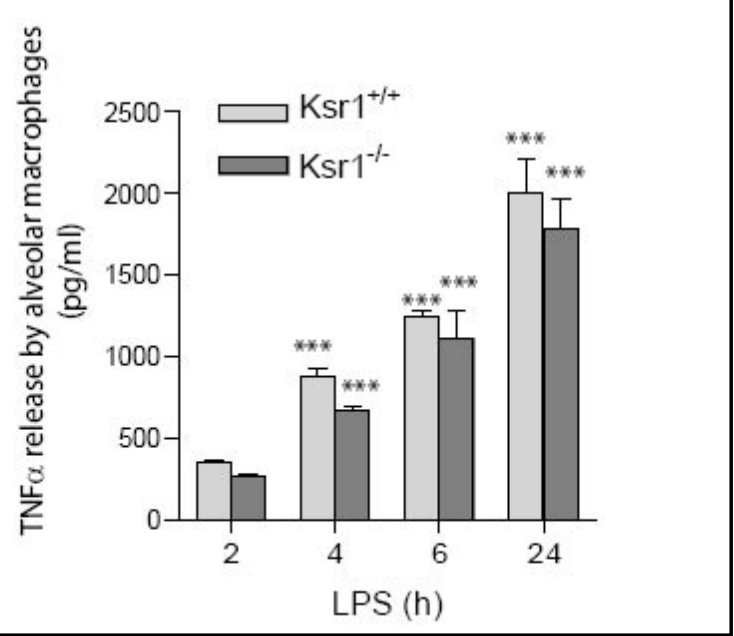

Role of Ksr1 in the activation of the Raf-1/MEK/ERK pathway in alveolar macrophages

Next, we aimed to determine whether Ksr1 is involved in ERK activation in alveolar macrophages. As shown in Fig. 5, ERK activation upon LPS stimulation was detected in both $\mathrm{Ksr}^{+/+}$and $\mathrm{Ksr}^{\%}{ }^{-}$cells, and maximum levels were reached at approximately $30 \mathrm{~min}$. To test for a role of Raf-1 in ERK activation upon LPS exposure, alveolar macrophages were pretreated with GW5074, a Raf-1 inhibitor, for 20 min after which LPS-induced ERK phosphorylation was determined. GW5074 abolished LPS-induced ERK phosphorylation. Pretreatment of the cells with a MEK inhibitor, U0126, also abolished LPS-induced ERK phosophorylation (data not shown). Next, we examined whether Raf- 1 is activated by Src-like tyrosine kinases. Pretreatment of cells with the Src inhibitor PP2 blocked LPS-induced ERK phosphorylation. Taken together, these findings indicate that LPS-induced Raf-1/MEK/ERK activation is mediated by Src-like tyrosine kinase-dependent and Ksr1-independent mechanisms.

\section{Discussion}

The present study demonstrates that Ksr1 is not necessary for the expression of inflammatory mediators, the infiltration of PMNs, and vascular leakage in response to LPS in a murine model of acute lung injury. In alveolar macrophages, Ksr1 is not involved in Raf- 
Fig. 5. Src kinase but not Ksr1 is involved in LPSinduced ERK activation in alveolar macrophages. Alveolar macrophages isolated from wild-type or Ksr1-deficient mice were treated with LPS (100 ng/ $\mathrm{mL}$ ) for the indicated time, and ERK activation was evaluated by Western blot analysis of phosphorylated isoforms p44 ERK1 (upper band) and p42 ERK2 (lower band). Alveolar macrophages were also pretreated with Raf-1 inhibitor GW-5074 $(10 \mu \mathrm{M})$ or Src inhibitor PP2 $(10 \mu \mathrm{M})$ for $15 \mathrm{~min}$. Total ERK1 and ERK2 were probed asaloadingcontrol.Shownare representativeblots from four independent experiments. Abbreviations: Ksr1, kinase suppressor of Ras-1; LPS, lipopolysaccharide.

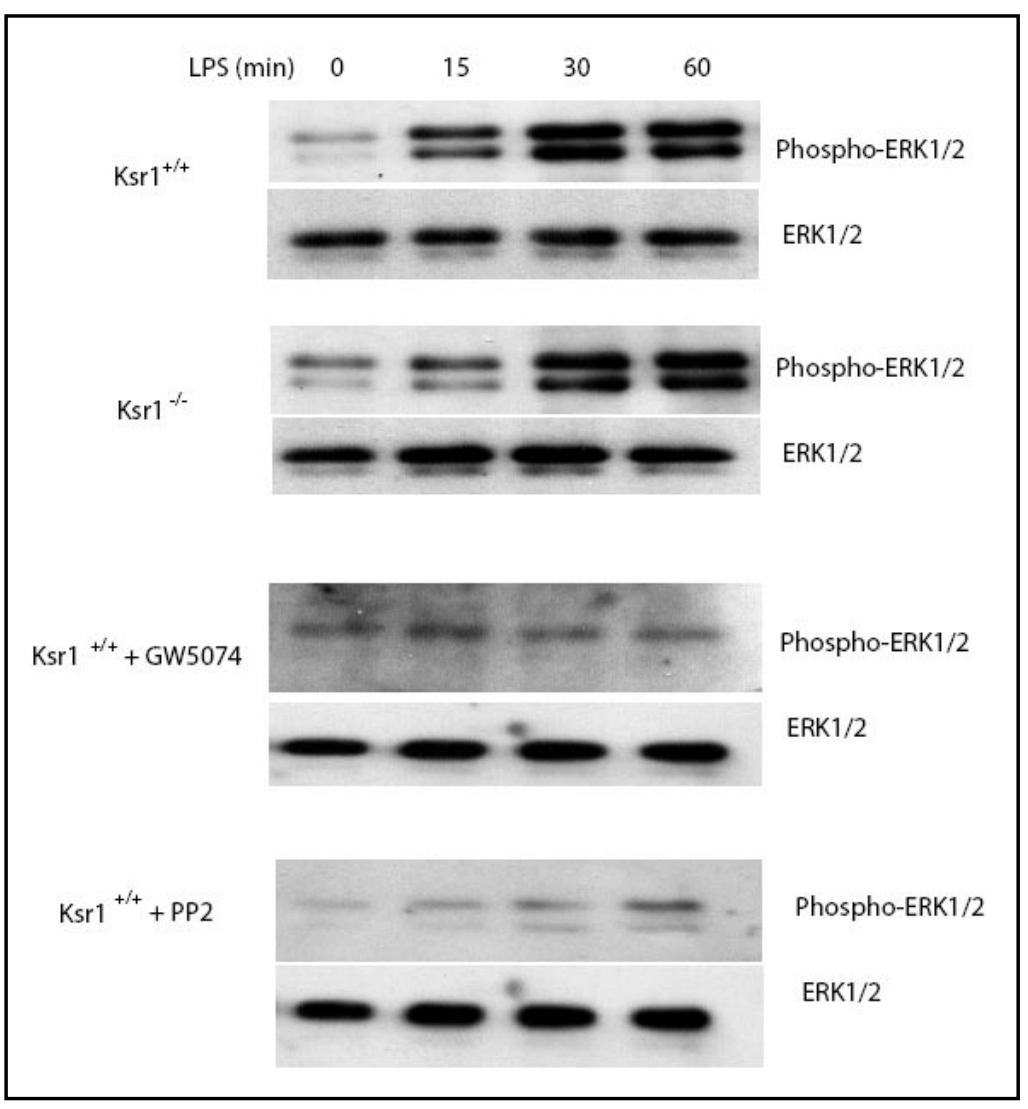

1/MEK/ERK signaling in response to LPS. Furthermore, Ksr1 plays no role in LPS-induced $\mathrm{NF} \kappa \mathrm{B}$ activation and cytokine production in these lung macrophages.

Previous studies from our group demonstrated a central role of Ksr1 in the host response to pulmonary P. aeruginosa infections [10]. These studies revealed that Ksr1 functions to coordinate heat-shock proteins and NO-synthases to release NO and NO-radicals upon $P$. aeruginosa infection. Ksr1-deficient mice were unable to kill the bacteria and to remove them from the lung resulting in severe pneumonia, sepsis and death of Ksr1-deficient mice infected with P. aeruginosa. The present study reveal the surprising result that LPS molecules, one of the major pathogenic factors of gram-negative bacteria, does not signal via Ksr1.

Recent studies have demonstrated that Ksr1 regulates the functions of immune cells in vitro and regulates immune responses in vivo. Fusselo and colleagues demonstrated that proinflammatory cytokine-induced ERK activation is reduced in macrophages derived from Ksr1-deficient bone marrow. The deficiency of Ksr1 results in resistance to joint damage in an antiserum-induced arthritis model, which requires only the innate immune system for pathogenesis. Thus, the action of Ksr1 in mice with antiserum-induced arthritis is attributed to its role in the positive regulation of ERK activation [8]. Recently, Lin and colleagues reported that Ksr1 functions as a MAPK scaffold protein that regulates the threshold required for ERK activation in T cells without affecting the nature of the response; they suggested that KSR1 plays a central role in determining the sensitivity of T-cell responses [20]. Thus, these studies suggest that Ksr1 may be involved in regulating the innate immune response in the lung by modulating ERK activation in lung macrophages.

Our findings, in contrast, show that Ksr1 plays a redundant role in a murine model of LPS-induced ALI. In the present study we demonstrated that LPS activates freshly isolated alveolar macrophages to produce cytokines. Ksr1 deficiency impairs neither nuclear translocation of transcription factor NFKB nor expression of inflammatory cytokine TNF $\alpha$. 
In summary, Ksr1 deficiency does not alter the main characteristics of LPS-induced ALI in the mouse lung, including neutrophil infiltration and accumulation, protein extrusion, and cytokine expression.

In cultured macrophages derived from mouse bone marrow, Fusselo and colleagues demonstrated that LPS-induced ERK activation is reduced in the absence of Ksr1 expression; this finding suggests that Ksr1 promotes the MEK/ERK signaling pathway [8, 19, 21, 22]. However, in the present study, we did not find a role of Ksr1 in LPS-induced ERK activation in lung macrophages. First, LPS stimulates ERK phosphorylation in both Ksr1-deficent and Ksr1-proficient lung macrophages in a similar pattern; this finding excludes a role for Ksr1 as a regulator in LPS-induced ERK activation of alveolar macrophages. Second, the blockade of Src-like tyrosine kinases or Raf-1 inhibits LPS-induced ERK activation; this inhibition suggests that the Raf-1/MEK/ERK signaling pathway is present and activated by Src-like tyrosine kinases/Raf-1-dependent mechanisms, which do not require Ksr1 as a scaffold. This finding is in line with previous in vivo findings showing that the inhibition of Src-like tyrosine kinases attenuates LPS-induced ALI in mice [23]. Taken together, our findings support the view that Ksr1 is not required for LPS-induced ERK activation in freshly isolated alveolar macrophages. LPS-induced activation of the MEK/ERK pathway is mediated, at least partially, through Src-like tyrosine kinases/Raf-1.

In conclusion, our results demonstrate that Ksr1 does not play a role in LPS-induced ERK activation in lung alveolar macrophages and does not contribute to the development of ALI induced by LPS.

\begin{abstract}
Abbreviations
Kinase suppressor of ras-1 (Ksr1); Lipopolysaccharides (LPS); Acute lung injury (ALI).
\end{abstract}

\title{
Acknowledgments
}

This study was funded by German Research Foundation (DFG) grant Gu 335-16/1 and NIH grant 2RO1HLO75316-05.

\section{References}

1 Matthay MA, Zimmerman GA, Esmon C, Bhattacharya J, Coller B, Doerschuk CM, Floros J, Gimbrone MA Jr, Hoffman E, Hubmayr RD, Leppert M, Matalon S, Munford R, Parsons P, Slutsky AS, Tracey KJ, Ward P, Gail DB, Harabin AL: Future research directions in acute lung injury: summary of a National Heart, Lung, and Blood Institute working group. Am J Respir Crit Care Med 2003;167:1027-1035.

-2 Rubenfeld GD, Caldwell E, Peabody E, Weaver J, Martin DP, Neff M, Stern EJ, Hudson LD: Incidence and outcomes of acute lung injury. N Engl J Med 2005;353:1685-1693.

3 Downward J: KSR: a novel player in the RAS pathway. Cell 1995;83:831-834.

4 Zhang Y, Yao B, Delikat S, Bayoumy S, Lin XH, Basu S, McGinley M, Chan-Hui PY, Lichenstein H, Kolesnick R: Kinase suppressor of Ras is ceramide-activated protein kinase. Cell 1997;89:63-72.

5 Nguyen A, Burack WR, Stock JL, Kortum R, Chaika OV, Afkarian M, Muller WJ, Murphy KM, Morrison DK, Lewis RE, McNeish J, Shaw AS: Kinase suppressor of Ras (KSR) is a scaffold which facilitates mitogenactivated protein kinase activation in vivo. Mol Cell Biol 2002;22:3035-3045.

6 Lozano J, Xing R, Cai Z, Jensen HL, Trempus C, Mark W, Cannon R, Kolesnick R: Deficiency of kinase suppressor of Ras1 prevents oncogenic ras signaling in mice. Cancer Res 2003;63:4232-4238. 


\section{Cellular Physiology Cell Physiol Biochem 2012;30:905-914 \begin{tabular}{l|l} 
Dol: 10.1159/000341468 & \\
and Biochemistry 2012 S. Karger AG, Basel
\end{tabular} \\ Li/Gulbins/Zhang: Ksr1 in LPS-Induced Acute Lung Injury}

7 Laurent MN, Ramirez DM, Alberola-Ila J: Kinase suppressor of Ras couples Ras to the ERK cascade during T cell development. J Immunol 2004;173:986-992.

8 Fusello AM, Mandik-Nayak L, Shih F, Lewis RE, Allen PM, Shaw AS: The MAPK scaffold kinase suppressor of Ras is involved in ERK activation by stress and proinflammatory cytokines and induction of arthritis. J Immunol 2006;177:6152-6158.

-9 Kolch W: Meaningful relationships: the regulation of the Ras/Raf/MEK/ERK pathway by protein interactions. Biochem J 2000;351 Pt 2:289-305.

-10 Zhang Y, Li X, Carpinteiro A, Goettel JA, Soddemann M, Gulbins E: Kinase suppressor of Ras-1 protects against pulmonary Pseudomonas aeruginosa infections. Nat Med 2011;17:341-346.

-11 Sibille Y, Reynolds HY: Macrophages and polymorphonuclear neutrophils in lung defense and injury. Am Rev Respir Dis 1990;141:471-501.

12 Kooguchi K, Hashimoto S, Kobayashi A, Kitamura Y, Kudoh I, Wiener-Kronish J, Sawa T: Role of alveolar macrophages in initiation and regulation of inflammation in Pseudomonas aeruginosa pneumonia. Infect Immun 1998;66:3164-3169.

-13 Lomas-Neira J, Chung CS, Perl M, Gregory S, Biffl W, Ayala A: Role of alveolar macrophage and migrating neutrophils in hemorrhage-induced priming for ALI subsequent to septic challenge. Am J Physiol Lung Cell Mol Physiol 2006;290:L51-L58.

14 Farley KS, Wang LF, Razavi HM, Law C, Rohan M, McCormack DG, Mehta S: Effects of macrophage inducible nitric oxide synthase in murine septic lung injury. Am J Physiol Lung Cell Mol Physiol 2006;290:L1164L1172.

15 Rowe SJ, Allen L, Ridger VC, Hellewell PG, Whyte MK: Caspase-1-deficient mice have delayed neutrophil apoptosis and a prolonged inflammatory response to lipopolysaccharide-induced acute lung injury. J Immunol 2002;169:6401-6407.

-16 Reinehr R, Sommerfeld A, Keitel V, Grether-Beck S, Häussinger D: Amplification of CD95 activation by caspase 8-induced endosomal acidification in rat hepatocytes. J Biol Chem 2008;283:2211-2222.

17 Reutershan J, Chang D, Hayes JK, Ley K: Protective effects of isoflurane pretreatment in endotoxin-induced lung injury. Anesthesiology 2006;104:511-517.

18 Yan F, John SK, Polk DB: Kinase suppressor of Ras determines survival of intestinal epithelial cells exposed to tumor necrosis factor. Cancer Res 2001;61:8668-8675.

19 Yan F, John SK, Wilson G, Jones DS, Washington MK, Polk DB: Kinase suppressor of Ras-1 protects intestinal epithelium from cytokine-mediated apoptosis during inflammation. J Clin Invest 2004;114:1272-1280.

20 Lin J, Harding A, Giurisato E, Shaw AS: KSR1 modulates the sensitivity of mitogen-activated protein kinase pathway activation in T cells without altering fundamental system outputs. Mol Cell Biol 2009;29:20822091.

-21 Kim M, Yan Y, Kortum RL, Stoeger SM, Sgagias MK, Lee K, Lewis RE, Cowan KH: Expression of kinase suppressor of Ras1 enhances cisplatin-induced extracellular signal-regulated kinase activation and cisplatin sensitivity. Cancer Res 2005;65:3986-3992.

-22 Kolch W: Coordinating ERK/MAPK signalling through scaffolds and inhibitors. Nat Rev Mol Cell Biol 2005;6:827-837.

23 Lee HS, Moon C, Lee HW, Park EM, Cho MS, Kang JL: Src tyrosine kinases mediate activations of NF- $\kappa B$ and -integrin signal during lipopolysaccharide-induced acute lung injury. J Immunol 2007;179:7001-7011. 